\title{
RAPID SYNTHESIS OF GOLD NANO-PARTICLES USING PULSE WAVED POTENTIAL IN A NON-AQUEOUS ELECTROLYTE
}

\begin{abstract}
Rapid synthesis of gold nanoparticles (AuNPs) by pulsed electrodeposition was investigated in the non-aqueous electrolyte, 1-ethyl-3-methyl-imidazoliumbis(trifluoro- methanesulfonyl)imide ([EMIM]TFSI) with gold trichloride ( $\left.\mathrm{AuCl}_{3}\right)$. To aid the dissolution of $\mathrm{AuCl}_{3}$, 1-ethyl-3-methyl-imidazolium chloride ([EMIM]Cl) was used as a supporting electrolyte in [EMIM]TFSI. Cyclic voltammetry experiments revealed a cathodic reaction corresponding to the reduction of gold at $-0.4 \mathrm{~V}$ vs. Pt-QRE. To confirm the electrodeposition process, potentiostatic electrodeposition of gold in the non-aqueous electrolyte was conducted at $-0.4 \mathrm{~V}$ for $1 \mathrm{~h}$ at room temperature. To synthesize AuNPs, pulsed electrodeposition was conducted with controlled duty factor, pulse duration, and overpotential. The composition, particle-size distribution, and morphology of the AuNPs were confirmed by field-emission scanning electron microscopy (FE-SEM), energy-dispersive spectroscopy (EDS), and transmission electron microscopy (TEM). The electrodeposited AuNPs were uniformly distributed on the platinum electrode surface without any impurities arising from the non-aqueous electrolyte. The size distribution of AuNPs could be also controlled by the electrodeposition conditions.

Keywords: gold, nanoparticle, non-aqueous electrolyte, pulse wave, catalyst
\end{abstract}

\section{Introduction}

Gold has outstanding chemical stability and is a good conductor of both heat and electricity. Because of these characteristics, gold has been a vital material for today's electronic industry. In the macroscale, gold does not absorb visible light at $530 \mathrm{~nm}$, however nano-sized gold particles experience the plasmon phenomenon which strongly absorbs visible light. Thus, the nano-sized gold has varying electrical and optical properties, dependent upon size and shape of the particles [1-5]. According to research conducted by Haruta, et al. in 1989, macroscale gold is catalytically inactive, whereas nano-sized gold is an effective catalyst in the reactions such as oxidation of carbon monoxide $(\mathrm{CO})$ and hydrogen $\left(\mathrm{H}_{2}\right)$, the reduction of nitrogen monoxide (NO), the water-gas shift reaction and the catalytic combustion of methanol $\left(\mathrm{CH}_{3} \mathrm{OH}\right)$ when the particles are supported by $\mathrm{TiO}_{2}$ or $\mathrm{Co}_{3} \mathrm{O}_{4}$ [6-7]. Because of these characteristics, AuNPs have been applied in various fields and applications such as catalysis, nano-bio sensors, nano-devices and nano-electrochemistry. They have particular importance in medical science, with gold expected to be applied to the research of rheumatoid arthritis and cancer treatment following combination with proteins, viruses and DNA [8-9].

Typically, gold nanoparticles are produced via the reduction of chloroauric acid $\left(\mathrm{H}\left[\mathrm{AuCl}_{4}\right]\right)$ using sodium citrate $\left(\mathrm{NaH}_{2}\right.$
$\left.\mathrm{C}_{6} \mathrm{H}_{5} \mathrm{O}_{7}\right)$ as the reducing agent. This method was reported by Turkevitch, et al. in 1951 and was able to produce AuNPs with a particle size of 10-20 nm of [10-11]. The AuNPs produced by this chemical method were able to be synthesized easily, however there are many problems stemming from this process such as acid waste, environmental pollution, and difficulties in controlling size and distribution of the particles [12-13].

Ionic liquids (ILs) are gradually receiving attention as an alternative solvent for electrochemical treatment of precious metals, due to their high thermal and chemical stabilities, low vapor pressures, relatively high ionic conductivities and wide electrochemical windows [14-15]. In addition, ILs have received considerable attention as eco-friendly solvents because they can be reused and do not generate volatile organic compounds and a side reaction such as $\mathrm{H}_{2}$ evolution [16-17].

Pulsed electrodeposition method applies a square-wave potential/current pulse that alternates between the applied potential/current and the resting state. In nanoparticle synthesis, pulsed electrodeposition has many advantages such as low cost, controllability, and a high purity product. In comparison with other electrolysis techniques, pulsed electrodeposition has the advantage of increasing catalytic efficiency due to an increased magnitude of nucleation sites on the working electrode [18]. The electrodeposition using a pulsed wave can be rapidly synthesized highly pure deposits in a non-aqueous electrolyte

\footnotetext{
SCHOOL OF ADVANCED MATERIALS SCIENCE AND ENGINEERING, KUMOH NATIONAL INSTITUTE OF TECHNOLOGY, 1 DAEHAK-RO, GUMI, GYUNGBUK, 39177 REPUBLIC OF KOREA

\# Corresponding author: cklee@kumoh.ac.kr
} 
because of no contamination by impurities from a side reaction such as hydrogen. It also makes AuNPs size control easier than conventional methods such as chemical vapor deposition and physical vapor deposition.

In this work, we report the electrochemical behavior of gold in a non-aqueous electrolyte for the synthesis of AuNPs $(<5 \mathrm{~nm})$ which show excellent catalytic performance [19]. The 1-ethyl-3-methyl-imidazoliumbis(trifluoromethanesulfonyl) imide ([EMIM]TFSI) was used as the non-aqueous electrolyte at room temperature due to its wide electrochemical window. 1-ethyl-3-methyl-imidazolium chloride ([EMIM]Cl) was used as a supporting electrolyte to aid dissolution $\mathrm{AuCl}_{3}$.

\section{Experimental}

A three-electrode electrochemical cell was used for the study and synthesis of AuNPs. Platinum wires (diameter $0.2 \mathrm{~mm}$ ) were used as working and reference electrode, while a platinum coil was used as the counter electrode. The cell and electrodes were cleaned prior to use in a sonicating bath using a solution of $\mathrm{H}_{2} \mathrm{SO}_{4}$ and $\mathrm{H}_{2} \mathrm{O}_{2}$ (1:1 vol.\%). $\mathrm{AuCl}_{3}$ (gold (III) chloride, SigmaAldrich) was used as the gold source for the AuNPs. For the electrolyte, a mixture solution of [EMIM]TFSI (C-TRI, Korea) and 0.1 M [EMIM]Cl (C-TRI, Korea) was used.

Cyclic voltammetry performed using a Potentiostat/Galvanostat (SP-240, Bio-Logic) at a scan rate of $10 \mathrm{mV} / \mathrm{s}$ over a potential range between $-1.5 \sim+1.5 \mathrm{~V}$ vs. Pt-QRE. Potentiostatic electrodeposition of gold was conducted at $-0.4 \mathrm{~V}$ vs. Pt-QRE for $1 \mathrm{~h}$. Pulsed electrodeposition was conducted while controlling duty factor, pulse duration, and overpotential to synthesize AuNPs. The average and standard deviation of the particles were determined by measuring 100 particle sizes on SEM image.

The morphology and chemical composition of the reduced gold and AuNPs were analyzed using a field emission scanning electron microscope (FE-SEM \& EDS, JEOL, JSM 6701F) and a transmission electron microscope (TEM, JEOL, JEM 2100).

\section{Results and discussion}

\subsection{Electrochemical reduction of gold in a non-aqueous electrolyte}

The oxidation and reduction behaviors of gold in a nonaqueous electrolyte were investigated at room temperature using cyclic voltammetry at a scan rate of $10 \mathrm{mV} / \mathrm{s}$. Fig. 1 shows the cyclic voltammograms of an electrolyte comprising [EMIM] TFSI, 0.1 M [EMIM]Cl with $0.05 \mathrm{M} \mathrm{AuCl}_{3}$ at platinum electrode. Two anodic peaks corresponding to gold oxidation are observed at potentials of $0 \mathrm{~V}$ and $0.5 \mathrm{~V}$ vs. Pt-QRE. The corresponding reduction peak is observed at a potential of $-0.4 \mathrm{~V}$ vs. Pt-QRE.

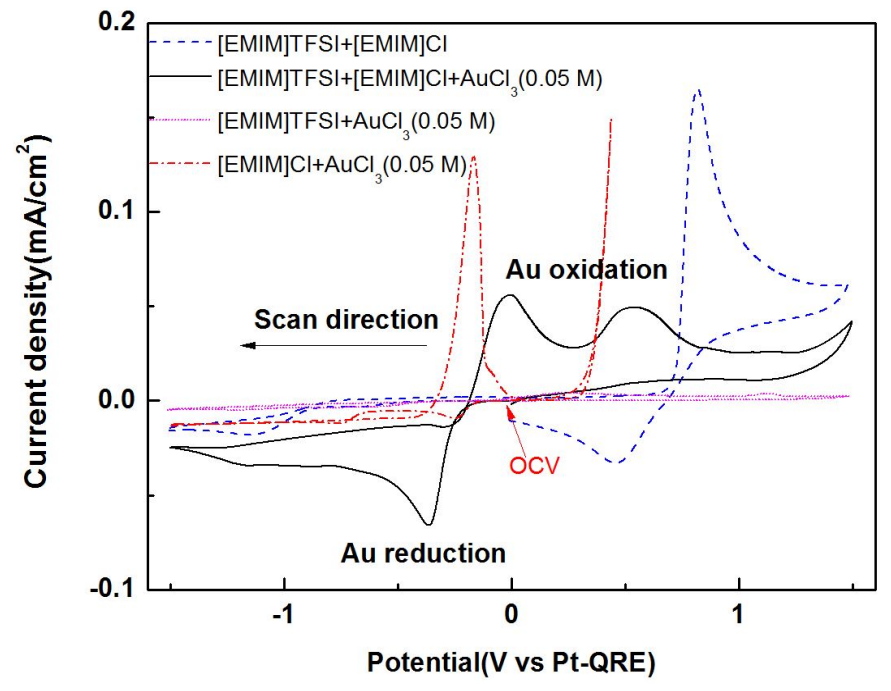

Fig. 1. Cyclic voltammograms of various electrolytes at a platinum working electrode(room temperature, $10 \mathrm{mV} / \mathrm{s}$ )

\subsection{Electrochemical reduction of gold in a non-aqueous electrolyte}

Electrodeposition of gold was conducted under potentiostatic conditions at $-0.4 \mathrm{~V}$ vs. Pt-QRE for $1 \mathrm{~h}$. Fig. 2 shows an FE-SEM image and EDS analysis of uniformly electrodeposited gold on a platinum electrode. No impurities were not detected by EDS analysis.

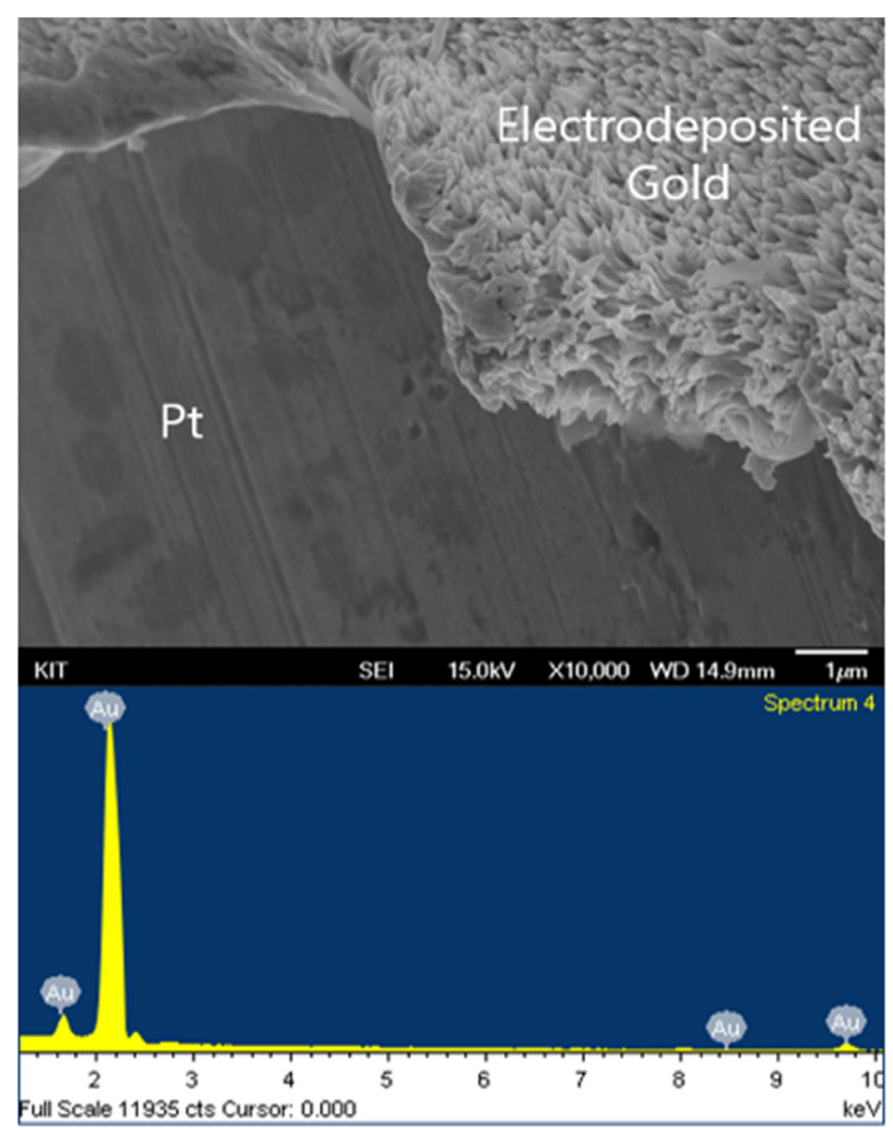

Fig. 2. FE-SEM and EDS analysis of gold electrodeposited on a platinum electrode potentiostatically at $-0.4 \mathrm{~V}$ for $1 \mathrm{~h}$ 


\subsection{Synthesis of gold nanoparticles using pulsed electrodeposition in a non-aqueous electrolyte}

\subsubsection{Effect of duty factor}

The duty factor in a pulsed electrodeposition experiment is defined by the formula:

$$
\text { Duty factor }=\frac{t_{1}}{t_{1}+t_{2}}
$$

$\left(t_{1}=\right.$ electrodeposition time, $t_{2}=$ rest time $)$

In order to investigate the effect of duty factor on synthesis of gold nanoparticles, electrodeposition time $\left(\mathrm{t}_{1}\right)$ was varied from 10 to $100 \mathrm{~ms}$. Fig. 3 shows the size distribution of AuNPs with variation of the duty factor, and demonstrates that an increase of the electrodeposition time from $10 \mathrm{~ms}$ to $100 \mathrm{~ms}$ resulted in a decrease of AuNPs size from $80 \mathrm{~nm}$ to $50 \mathrm{~nm}$. It is thought that the surface concentration of gold ion was not enough due to the low diffusion rate of gold ions in the non-aqueous electrolyte while the electrodeposition time increased, and nucleation should be dominant rather than crystal growth. We have thus shown that the size of AuNPs could be controlled by adjusting the duty factor of the electrodeposition experiment.

\subsubsection{Effect of pulse duration}

Fig. 4 shows the size distribution of AuNPs resulting from variation of the pulse duration from 10 to $60 \mathrm{~s}$. Notably, the size of AuNPs increased from $70 \mathrm{~nm}$ to $150 \mathrm{~nm}$ with increasing pulse duration due to the great influence of crystal growth relative to nucleation. Therefore, it is confirmed that AuNPs size above $70 \mathrm{~nm}$ are able to be controlled by adjusting pulse duration.

\subsubsection{Effect of overpotential}

To investigate the effect of overpotential on the synthesis of AuNPs, the overpotential of potentiostatic electrodeposition was controlled from -0.5 to $-3 \mathrm{~V}$ vs. Pt-QRE. Fig. 5 shows the size distribution of AuNPs and demonstrates that with an increase of the overpotential from -0.5 to $-3 \mathrm{~V}$ vs. Pt-QRE, the size of AuNPs decreased from $60 \mathrm{~nm}$ to $5 \mathrm{~nm}$, due to the increased influence of nucleation over crystal growth. Therefore, it is confirmed that AuNPs of size less than $60 \mathrm{~nm}$ are able to be controlled by adjusting the electrodeposition overpotential.

\subsubsection{Synthesis of $5 \mathrm{~nm}$ AuNPs}

The above experiments demonstrated a set of variables that determine the size of AnNPs growth in our chosen electrolyte. Thus, we were able to manipulate these conditions in order to synthesize AuNPs of size $\sim 5 \mathrm{~nm}$. Pulsed electrodeposition was performed with a duty factor of 0.5 , pulse duration of $5 \mathrm{~s}$, and

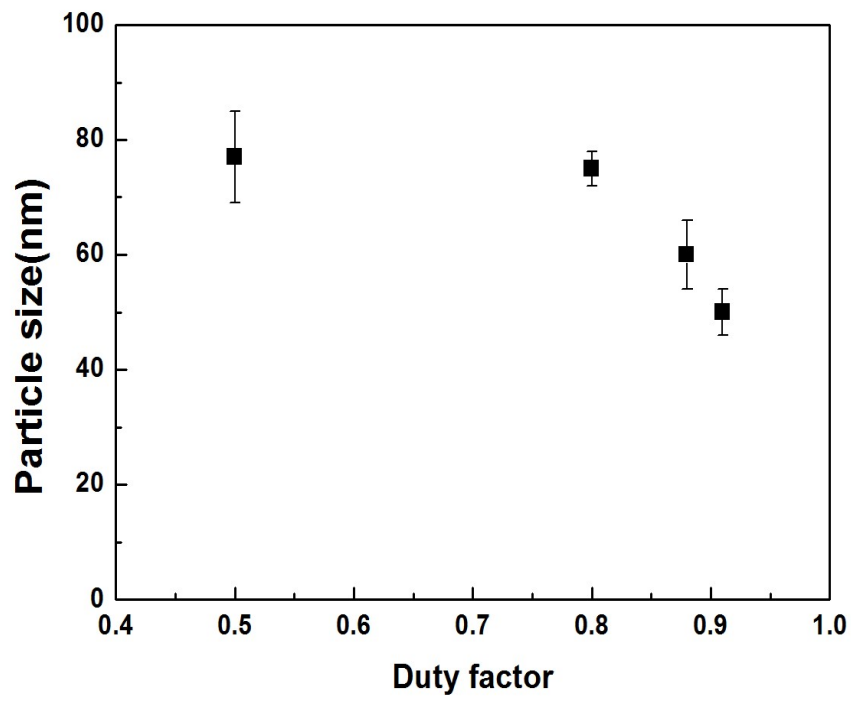

Fig. 3. Effect of duty factor on particle size of AuNPs by pulsed electrodeposition

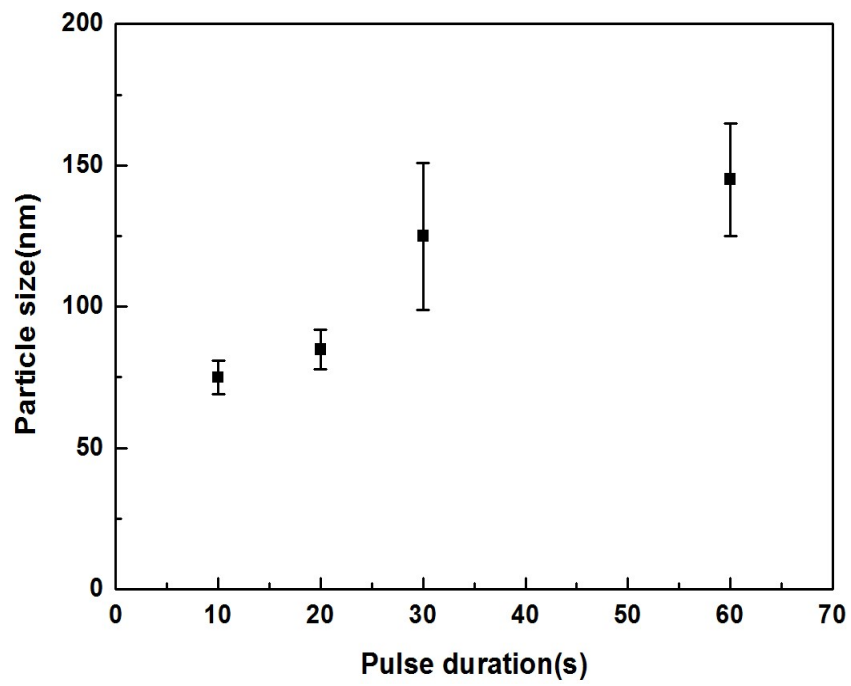

Fig. 4. Effect of pulse duration on particle size of AuNPs by pulsed electrodeposition

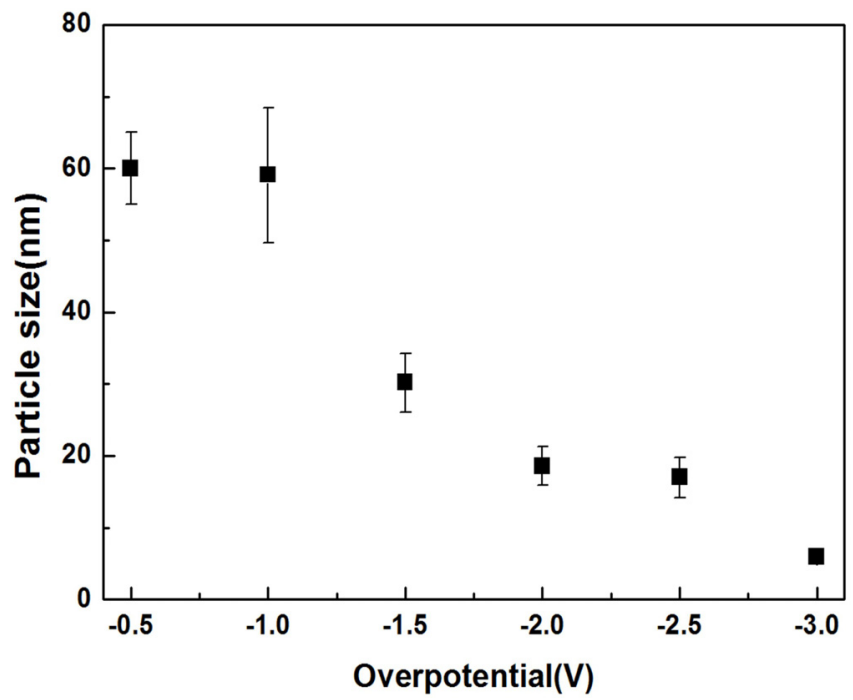

Fig. 5. Effect of overpotential on particle size of AuNPs by pulsed electrodeposition 
-3 V vs. Pt-QRE deposition overpotential in a non-aqueous electrolyte comprising [EMIM]TFSI, [EMIM]Cl, and $0.05 \mathrm{M}$ $\mathrm{AuCl}_{3}$. Following pulsed electrodepositon at these conditions, AuNPs having average size of $5.9 \mathrm{~nm}$ and standard deviation of $0.7 \mathrm{~nm}$ were prepared as shown in Fig. 6.

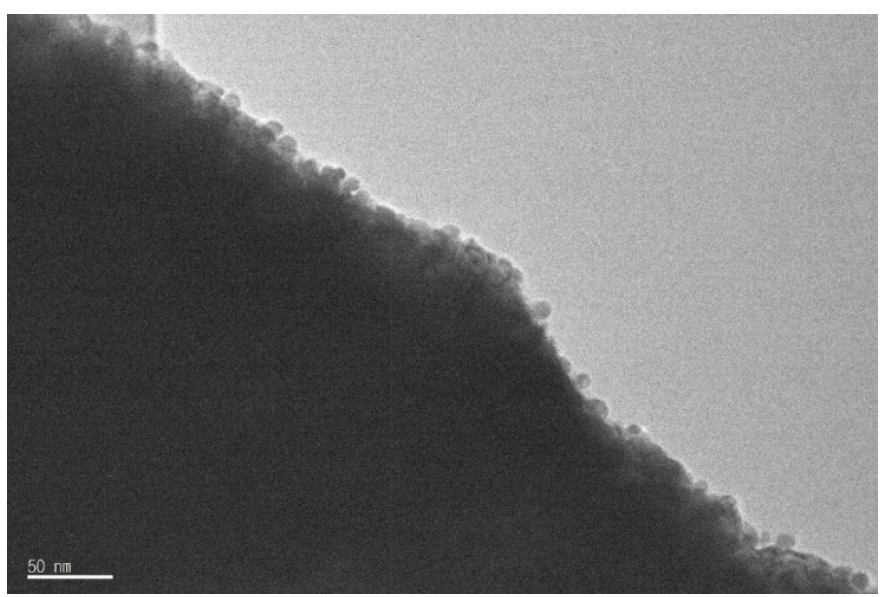

Fig. 6. TEM image of AuNPs synthesized by pulsed electrodeposition

\section{Conclusions}

In this study, the synthesis of size-controllable AuNPs was achieved using pulsed electrodeposition in a non-aqueous electrolyte at room temperature. The ionic liquid [EMIM]TFSI was used as the non-aqueous electrolyte due to its high ionic conductivity and low viscosity. However, due to the low solubility of $\mathrm{AuCl}_{3}$ in this ionic liquid, [EMIM]Cl was used as a supporting electrolyte. Cyclic voltammetry revealed a gold reduction event at $-0.4 \mathrm{~V}$ vs. Pt-QRE. After potentiostatic electrodeposition of gold at this potential for $1 \mathrm{~h}$, only pure gold was observed on the platinum electrode as confirmed by EDS analysis.

Pulsed electrodeposition was conducted with variation of the duty factor, pulse duration, and overpotential, and each factor was shown to control the size of the resulting AuNPs. Increase of duty factor and overpotential decreased the size of AuNPs, whereas AuNPs size increased with increasing pulse duration. AuNPs near to $5 \mathrm{~nm}$ in diameter sizes known for their high catalytic activity were thus able to be synthesized at $-3 \mathrm{~V}$ vs. Pt-QRE, with a duty factor of 0.5 and pulse duration of $5 \mathrm{~s}$. Consequently, we have shown that it is possible to reduce various sizes of AuNPs at an electrode through pulsed electrodeposition techniques.

\section{Acknowledgments}

This paper was supported by the Research Fund of the Kumoh National Institute of Technology.

\section{REFERENCES}

[1] S.I. Yang, Physics \& High Technology 15, 31 (2006).

[2] E.E. Connor, J. Mwamuka, A. Gole, C.J. Murphy, M.D. Wyatt, Small. 1, 325 (2005).

[3] P. Ghosh, G. Han, M. De, C.K. Kim, V.M. Rotello, Adv. Drug Deliver. Re. 60, 1307 (2008).

[4] P. Anger, P. Bharadwaj, L. Novotny, Phys. Rev. Lett. 96, 113002 (2006).

[5] X. Huang, M.A. El-Sayed, Journal of Advanced Research 1, 13 (2010).

[6] M.-C. Daniel and D. Astruc, Chem. Rev. 104, 293 (2004).

[7] M. Haruta, Cat. Tech. 6, 102 (2002).

[8] H.N. Kim, Thesis of PhD, Inha University, (2009).

[9] Y. Pan, S. Neuss, A. Leifert, et al., Small. 3, 1941 (2007).

[10] A.N. Shipway, E. Katz, I. Willner, Chem. Phys. Chem. 1, 18 (2000).

[11] J. Turkevitch, P.C. Stevenson, J. Hillier, Discuss. Faraday. Soc. 11, 55 (1951).

[12] V.V. Makarov, A.J. Love, O.V. Sinitsyna, S.S. Makarova, I.V. Yaminsky, M.E. Taliansky, N.O. Kalinina, Acta Nature 6, 35 (2014).

[13] S. Iravani, H. Korbekandi, S.V. Mirmohammadi, B. Zolfaghari, Research in Pharmaceutical Sciences 9, 385 (2014).

[14] Y. Lu, K. Korf, Y. Kambe, Z. Tu, L.A. Archer, Angewandte Chemie 53, 488 (2014).

[15] J.O. Lee, G.W. Park, J.S. Park, Y.J. Cho, C.K. Lee, International J. of Precision Engineering and Manufacturing 16, 1220 (2015).

[16] S. Zhang, N. Sun, X. He, X. Lu, and X. Zhang, J. Phys. Chem. Ref. Data 35, 1475 (2006).

[17] J.S. Park, Y.J. Jung, P. Kusumah, J.Y. Lee, K.J. Kwon, C.K Lee, Int. J. Mol. Sci. 15, 15320 (2014).

[18] J. Liu, C. Zhong, X. Du, J. Liu, et al, Electrochimica Acta 100, 164 (2013).

[19] B. Hvolbaek, T.V.W. Janssens, B.S. Clausen, H. Falsig, C.H. Christensen, J.K. Norskov, Nano Today 2, 14 (2007). 